DETERMINACION DE LA CONFIGURACION E-Z DE LOS ACIDOS FUMARICO Y MALEICO. UN EXPERIMENTO ORIENTADO A INCENTIVAR EL DESARROLLO DE LA INVESTIGACION CIENTIFICA EN ALUMNOS DE PREGRADO

\title{
Carlos Bustos
}

Instituto de Química - Universidad Austral de Chile - Casilla 567 - Valdivia - Chile

Guillermo Salgado, Rolando Martínez

Deutsche Schule - Casilla 129 - Correo 30 - Santiago - Chile

François Carrière

Université Pierre et Marie Curie - 4 Place Jussieu - 75252 Cedex 05 - Paris - France

\begin{abstract}
DETERMINATION OF THE $E$ - $Z$ FUMARIC AND MALEIC ACIDS CONFIGURATION. AN EXPERIMENT DESIGNED TO DEVELOP SCIENTIFIC RESEARCH ABILITIES IN UNDERGRADUATES STUDENTS. In this work we intend to eliminate the idea that laboratory exercises seem like cookbooks. That is, exercises shall be presented as a problematic situation. Based on observation and experimentation, the students should determine the $E-Z$ configuration of maleic and fumaric acids. The basis of this laboratory exercise is the acid-catalyzed isomerization of maleic acid to fumaric acid. Students are given the starting material, reagents and the experimental procedure. They are told that the starting material is a dicarboxylic acid containing a $\mathrm{C}=\mathrm{C}$ double bond of formula $\mathrm{C}_{4} \mathrm{H}_{4} \mathrm{O}_{4}$. Students determine melting points, solubilities, acidity and chromatographic patterns for both the starting material and the product, so that a configuration of each acid can be proposed. This type of experiment yields excellent results, because the students are left to deduce that maleic acid is less stable than fumaric acid. Additionally, they conclude that maleic acid is the " $Z$ " isomer and fumaric acid is the " $E$ " isomer. Finally, this laboratory exercise allows the students to develop simultaneously their critical-thinking skills with the respective laboratory techniques and not to see chemistry as recipes to be followed.
\end{abstract}

Keywords: demonstration experiments; stereochemistry; chemical education.

\section{INTRODUCCIÓN}

El desarrollo de actividades de laboratorio es una oportunidad ideal para poner en práctica el Método Científico y, de este modo, obtener respuestas acertadas para un problema planteado. Sin embargo, frecuentemente, los objetivos fundamentales buscados en el desarrollo de un experimento no son alcanzados, la principal razón es que en la literatura científica, muchos de los experimentos se dan a conocer como simples recetas de cocina ${ }^{1}$. Una investigación publicada hace algún tiempo ${ }^{2}$, plantea la idea de realizar las demostraciones experimentales de manera tal que el estudiante desarrolle su espíritu crítico. Por este motivo se ha diseñado esta actividad, cuyo interés es mostrar como se lleva a cabo un experimento bajo el rigor del Método Científico y, al mismo tiempo, despertar el interés de los estudiantes por la investigación científica.

Una reacción que reúne los requisitos para realizar este tipo de demostración experimental, es la isomerización del ácido maleico a ácido fumárico catalizada por un ácido mineral $^{3}$, Fig. 1. Las ventajas principales que presenta esta reacción son dos: i) es una reacción lo suficientemente rápida y ii) debido a que los componentes A y $\mathbf{B}$ tienen propiedades físicas marcadamente distintas, la medición de algunas de estas propiedades, permite establecer hipótesis relativas a la estabilidad, estructura y/o configuración de las moléculas involucradas. Simultáneamente, el estudiante aprende y aplica una conjunto de técnicas de uso habitual en el laboratorio.

e-mail: salgado@interaccess.cl<smiles>O=C(O)/C=C/C=C/C(=O)OCC(=O)O</smiles>

Figura 1. Reacción de isomerización del ácido maleico a ácido fumárico, catalizada por un ácido mineral.

Para enfrentar los objetivos, los estudiantes reciben todo el material de laboratorio necesario, los reactivos y el procedimiento experimental. Como información previa ellos, solamente, saben que el compuesto de partida, A, tiene la fórmula $\mathrm{C}_{4} \mathrm{H}_{4} \mathrm{O}_{4}$. Por otro lado, aplicando las reglas de la química orgánica, ellos determinan el número de insaturaciones, luego, concluyen que la molécula es un ácido dicarboxílico y que, además, contiene un doble enlace $\mathrm{C}=\mathrm{C}$. Luego, una vez realizada la síntesis, los estudiantes determinan el punto de fusión, la solubilidad relativa, la acidez relativa de las disoluciones, la reactividad de las disoluciones frente a magnesio y el patrón cromatográfico, del reactivo, A, y del producto, B. Finalmente, bajo la dirección del Profesor, el conjunto de observaciones obtenidas permiten al etudiante obtener una serie de inferencias conducentes a proponer, en último término, la configuración de los isómeros estudiados.

\section{CONOCIMIENTOS PREVIOS}

Para llevar a cabo esta demostración experimental, se requiere que los estudiantes hayan cursado al menos un semestre de Química Orgánica en un Liceo. Se recomienda que el 
Profesor o Instructor realice una actividad de pre-laboratorio, para recordar o explicar en forma general todo lo relativo a las reglas para calcular el número de insaturaciones, las propiedades relativas a los isómeros $\boldsymbol{E}-\boldsymbol{Z}$, las interacciones por puente de hidrógeno y los fundamentos de las técnicas de laboratorio involucradas.

La cantidad de material de partida, A, usado en la síntesis dependerá del tamaño o volumen del material fungible disponible en el laboratorio, por lo cual, es posible diseñar la síntesis en escala semi-micro ${ }^{4}$, distinta de la aquí usada, sin que haya diferencias en los resultados. Por otro lado, en común acuerdo con el Profesor, los estudiantes, pueden planificar el experimento, de modo que, el trabajo se desarrolle en un máximo de cuatro horas pedagógicas de laboratorio, lo cual es equivalente a una sesión de $180 \mathrm{~min}$. La misma demostración experimental, proporciona buenos resultados con estudiantes que cursan su primera asignatura de Química Orgánica en la Universidad.

\section{PARTE EXPERIMENTAL}

\section{Procedimiento}

1: Transformación del ácido $\boldsymbol{A}$ en el ácido $\boldsymbol{B}$.

(a) En un matraz Erlenmeyer de $100 \mathrm{~mL}$ se introducen $6.00 \mathrm{~g}$ del compuesto de partida, etiquetado como ácido $\mathbf{A}$, seguido de $10.0 \mathrm{~mL}$ de agua destilada. La mezcla se calienta en un baño de agua, hasta completar disolución del sólido.

(b) Luego, cuidadosamente, a la disolución se agregan $15.0 \mathrm{~mL}$ de $\mathrm{HCl}$ concentrado, $\sim 12.5 \mathrm{~mol} / \mathrm{L}$. El matraz Erlenmeyer se cubre con un vidrio reloj y, la mezcla en reacción, se calienta a ebullición durante unos $3 \mathrm{~min}$, bajo campana, hasta observar la precipitación de un sólido.

(c) A continuación, con la ayuda de un baño de agua fría, se permite que la mezcla alcance la temperatura ambiente.

(d) El sólido se filtra por succión y se lava con pequeñas porciones de agua destilada y fría.

(e) El sólido obtenido se coloca sobre un vidrio reloj, se deja secar al aire o se presiona sobre papel filtro. Se puede, también, mantener en el interior de una estufa a $90-100{ }^{\circ} \mathrm{C}$, durante el transcurso de la sesión de trabajo. Este ácido se etiqueta como ácido $\mathbf{B}$.

\section{2: Análisis y Comparación de los Isómeros.}

(a) Determinación de la solubilidad relativa: En tubos de ensayo separados, se compara la solubilidad del ácido A y del ácido $\mathbf{B}$ disolviendo $1.00 \mathrm{~g}$ de cada uno de ellos en $10.0 \mathrm{~mL}$ de agua destilada

(b) Determinación de los puntos de fusión: El punto de fusión de los ácidos A y $\mathbf{B}$ se determina utilizando un aparato de punto de fusión o un aparato de Thiele.

(c) Determinación de la acidez y la reactividad con magnesio: En tubos de $\mathrm{f}>$ kayo separados, se prepara una disolución de A y otra de $\mathbf{B}$, disolviendo $0.10 \mathrm{~g}$ de cada ácido en $20 \mathrm{~mL}$ de agua destilada. A continuación, usando Papel Indicador Universal, se mide el pH de cada disolución. Luego, a cada tubo, se agrega $3.0 \mathrm{~cm}$ de cinta de magnesio, se anota la reacción ocurrida y se establece una relación entre el $\mathrm{pH}$ de la disolución con el nivel de burbujeo del gas hidrógeno.

(d) Determinación del patrón cromatográfico: Se preparan tres disoluciones diluidas, conteniendo A puro, B puro y otra que contenga una mezcla de $\mathbf{A}$ y $\mathbf{B}$. Para ello, se disuelve una pequeña cantidad, $\sim 50 \mathrm{mg}$ del ácido $\mathbf{A}$ o $\mathbf{B}$ puros $\mathrm{y}$ $\sim 25 \mathrm{mg}$ de cada uno de ellos en la mezcla, en $\sim 1.0 \mathrm{~mL}$ de etanol o acetona, respectivamente. A continuación, usando un capilar de vidrio, se aplica cada disolución sobre una placa de Sílica Gel, GF-254, para cromatografía en capa fina, de $5 \times 10 \mathrm{~cm}$. Se espera unos 5 min para que el solvente se evapore y se registra el cromatograma, usando como fase móvil una disolución acuosa de ácido acético al
15\%. El cromatograma, se revela con ácido sulfúrico, con radiación UV, o con vapores de yodo, luego, se marca y se obtienen los respectivos valores de $\mathrm{R}_{\mathrm{f}}$.

Anote todas las observaciones y las mediciones registrándolas en la siguiente Tabla:

\begin{tabular}{c|c|c|c|c} 
Acido & \multicolumn{1}{c}{$\begin{array}{c}\text { Punto de } \\
\text { fusión }\left({ }^{\circ} \mathrm{C}\right)\end{array}$} & $\begin{array}{c}\text { Solubilidad } \\
\text { en agua }\end{array}$ & \multicolumn{1}{c}{$\begin{array}{c}\text { Acidez, } \\
\mathrm{pH}\end{array}$} & $\mathrm{R}_{\mathrm{f}}$ \\
$\mathrm{A}$ & & & & \\
\hline B & & & & \\
\hline
\end{tabular}

\section{METODODOLOGIA PARA ENFRENTAR EL ANALISIS Y DISCUSION DE LOS RESULTADOS}

Dado que los estudiantes conocen la fórmula molecular, se debe conservar en secreto la simbología, $\mathbf{A}=$ ácido maleico y $\mathbf{B}=$ ácido fumárico, para evitar la posibilidad que ellos puedan investigar en sus textos de estudio la clave del problema. Al final del experimento, ellos deben elaborar las inferencias necesarias para descubrir la información que falta. Para el análisis y discusión de los resultados, es conveniente realizar una sesión de post-laboratorio y centrar la discusión considerando un esquema de trabajo como el que se muestra a continuación:

1. Basándose en las diferencias de puntos de fusión, solubilidad relativa, acidez relativa de las disoluciones y los valores de $\mathrm{R}_{\mathrm{f}}$, explicar las diferencias de comportamiento químico observadas en los ácidos $\mathbf{A}$ y $\mathbf{B}$.

2. Usando modelos moleculares de los isómeros $\boldsymbol{E}-\boldsymbol{Z}$, dar una respuesta satisfactoria sobre la estructura de los dos ácidos.

3. Sabiendo que uno de estos ácidos puede perder una molécula de agua, mediante calentamiento, para originar el correspondiente anhídrido, determinar cuál de los ácidos es susceptible de presentar este comportamiento.

Resultados obtenidos por los estudiantes:

\begin{tabular}{ccccc} 
Acido & $\begin{array}{c}\text { Punto de } \\
\text { fusión, }{ }^{\circ} \mathrm{C}\end{array}$ & $\begin{array}{c}\text { Solubilidad } \\
\text { en } \mathrm{H}_{2} \mathrm{O}\end{array}$ & $\begin{array}{c}\text { Acidez*, } \\
\mathrm{pH}\end{array}$ & $\mathrm{R}_{\mathrm{f}}$ \\
\hline $\mathrm{A}$ & $130^{\circ} \mathrm{C}$ & alta & 1.6 & 0.64 \\
$\mathrm{~B}$ & $302^{\circ} \mathrm{C}$ & baja & 3.5 & 0.70 \\
\hline
\end{tabular}

*Al adicionar cinta de magnesio a las disoluciones de A y B, se observa mayor nivel de burbujeo de $\mathrm{H}_{2}$ en la disolución que contiene el ácido A.

\section{CONCLUSIONES}

La realización de este experimento produjo excelentes resultados. Los estudiantes obtuvieron, sin problema, conclusiones tales como: i) el ácido fumárico es termodinámicamente más estable que el ácido maleico, ii) la estabilidad relativa está relacionada con la estereoquímica alrededor del doble enlace $\mathrm{C}=\mathrm{C}$, iii) el ácido $\mathbf{A}$, isómero $\boldsymbol{Z}$, corresponde al ácido maleico, y el ácido $\mathbf{B}$, isómero $\boldsymbol{E}$, corresponde al ácido fumárico iv) la mayor solubilidad, acidez y reactividad con magnesio, mostrada por las disoluciones acuosas de ácido maleico, respecto del ácido fumárico, están relacionadas con la extensión de la reacción de hidrólisis. Dicha reacción alcanza mayor extensión como consecuencia de la interacción por puente de hidrógeno intramolecular de uno de los dos protones ionizables del ácido maleico, aumentando la deficiencia electrónica en el otro, quien trata de neutralizar el efecto interactuando con los electrones no enlazantes de las moléculas de solvente, $\mathrm{H}_{2} \mathrm{O}$ v) el $\mathrm{R}_{\mathrm{f}}$ del 
ácido maleico es menor que el del ácido fumárico, debido a que el ácido acético contenido en la fase móvil, perturba los puentes de hidrógeno intramoleculares, de modo que, el ácido maleico aumenta su afinidad por la fase estacionaria y vi) la disposición espacial de los grupos carboxilos del ácido maleico, permiten que pueda perder una molécula de agua para generar el respectivo anhídrido, mediante calentamiento.

Las conclusiones anteriores fueron elaboradas en la sesión de post-laboratorio, guiada por el Profesor, donde los estudiantes confrontaron sus resultados e ideas y, en algunos casos, aclararon conceptos. Además, ellos fueron capaces de aplicar sus conocimientos para arriesgar hipótesis relativas a situaciones nuevas, $\boldsymbol{v}$. $g r$. el mecanismo de reacción. Los principales beneficios de esta demostración experimental son, por una parte, que los estudiantes, bajo la dirección del Profesor, juegan un rol activo $y$, por otra, aprenden variadas técnicas de laboratorio.

Finalmente, el desarrollo del experimento satisface a los estudiantes porque activa su espíritu crítico, permite apaciguar el pánico o la fobia a la química, despierta el interés por la investigación científica y comprenden que un procedimiento químico no es comparable con una receta de cocina.

\section{AGRADECIMIENTOS}

Los autores agradecen al Dr. B. Wojciechowski y al Dr. César López V., por las sugerencias para la elaboración del manuscrito. Además, se agradece a la Dirección de Investigación y Desarrollo de la Universidad Austral de Chile. (D.I.D. UACh, P. S/98/26).

\section{REFERENCIAS}

1. Pickering. M.; J. Chem. Educ. 1988, 65,143.

2. Wojciechowski B. J., Deal. S. T.; J. Chem. Educ. 1996, $73,85$.

3. Meek. J. S.; J. Chem. Educ. 1975, 52, 541.

4. A. Vogel's. Elementary Practical Organic Chemistry 1: Preparations. Longman, London, England, 1980, Ch. IV(38), p. 233. 